\title{
Long term follow-up of the patients with pelvic organ prolapse after the mesh implantation using strict indication criteria
}

\author{
Papcun P, Krizko M Jr, Spodniakova B, Redecha M, Gabor M, Ferianec V, Holly I \\ 2nd Department of Obstetrics and Gynecology, Medical Faculty at the Comenius University, and University \\ Hospital in Ruzinov, Bratislava, Slovakia. ivanhollyba@gmail.com
}

\begin{abstract}
Objectives: Transvaginal polypropylene mesh implantation is one of the techniques used for pelvic organ prolapse (POP) repair. The surgery outcomes depend on the indication criteria used. The aim of our study was to evaluate the outcomes of the mesh implantation using the strict indication criteria.

Patients and methods: In 47 women aged $61.7 \pm 8$.3 years with pelvic organ prolapse (POP-Q $\geq 2)$ and a history of other surgery in the pelvic region outcomes of the mesh implantation were evaluated for up to 7 years (range $1-7$ years).

Results: Forty six of 47 patients $(97.8 \%)$ had a successful mesh implantation (10 anterior, 22 posterior, 14 combined). Peroperative complications occurred in 3 of 47 patients $(6.4 \%)$. The anatomic cure (POP-Q $\leq 1)$ was achieved in $93.5 \%$ patients with mesh at 6 months after surgery. Any of the postoperative complications occurred in 16 of 46 women (34.8\%). Significantly lower risk of complications was found in the group aged over 65 years compared to the younger patients $(p=0.005)$.

Conclusion: This is the first study on the mesh implantation including women only with the history or other surgery in the pelvic region, achieving high anatomic success rate and low risk of complications. Thus, our data support the use of the strict indication criteria for this procedure (Tab. 2, Fig. 2, Ref. 14). Text in PDF www.elis.sk. Key words: pelvic organ prolapse, mesh, surgery, complications.
\end{abstract}

Pelvic floor disorders in women because of their high prevalence are one of the frequently discussed topics in gynecology. Pelvic organ prolapse is reported in up to $50 \%$ of parous women (1), and lifetime risk of undergoing pelvic reconstructive surgery around $11 \%$ (2). Nevertheless, the risk of recurrence of the prolapse after traditional prolapse surgery up to $58 \%$ has been reported (3). Therefore, other methods of pelvic organ prolapse have been searched. One of currently often used methods is the implantation a polypropylene mesh. Studies focused on perioperative morbidity and clinical outcomes using mesh implantation have yielded promising clinical outcomes (4). On the other hand, this therapeutic approach could have severe complications (e.g.

2nd Department of Obstetrics and Gynecology, Medical Faculty at the Comenius University, and University Hospital in Ruzinov, Bratislava, Slovakia

Address for correspondence: I. Holly, MD, PhD, 2nd Department of Obstetrics and Gynecology, Medical Faculty at the Comenius University, and University Hospital in Ruzinov, Ruzinovska 6, SK-826 06 Bratislava, Slovakia.

Phone: +421.2.48234630

Acknowledgements: We would like to thank Professor Holomán̆, MD, CSc. from the $2^{\text {nd }}$ Department of Obstetrics and Gynecology of the Medical Faculty at the Comenius University in Bratislava, and Juraj Stanik, MD, $\mathrm{PhD}$. from the $1^{\text {st }}$ Departmet of Pediatrics of the Medical Faculty at the Comenius University in Bratislava for the technical support. mesh exposure, infection, organ perforation, pain), especially using it in every woman with pelvic organ prolapse (5). Due to the FDA and Expert Committee Opinion, the pelvic organ prolapse vaginal mesh should be reserved for high-risk individuals in whom the benefit of mesh placement may justify the risk, such as individual with recurrent prolapse or with medical comorbidities (6).

In 2009, we have published a pilot study on 18 women using polypropylen mesh as a treatment of the pelvic organ prolapse (7). Currently, the aim of our study was: 1 . follow up of the 18 previously presented and further 29 patients with the pelvic organ prolapse up to 7 years after the mesh implantation, and 2. to compare our results using strict indication criteria for the surgery with other studies.

\section{Patients and methods}

\section{Patients}

In this prospective cohort study 47 patients with symptomatic pelvic organ prolapse were included at the 2 nd Department of Obstetrics and Gynecology in Bratislava, Slovakia since November 2006 to January 2013. The inclusion criteria were pelvic organ prolapse stage POP-Q $\geq 2$, and history of other surgery in the pelvic region in the past, e.g. hysterectomy or traditional surgery of the pelvic organ prolapse. The mean age at surgery was $61.7 \pm 8.3$ 
years. More than a half of the patients $(61.7 \%)$ had undergone previous traditional prolapse surgery, and 44/47 (93.6\%) had hysterectomy in the past.

\section{Clinical examination prior the mesh implantation}

A gynecological examination and subjective symptom assessments were performed prior the surgery. POP-Q quantification was used for the anatomical prolapse stage evaluation. Methods, definitions, and descriptions conformed to the standards recommended by the ICS (8).

\section{Surgery}

The mesh implantation was performed using the polypropylene transvaginal mesh kit (Prolift $\left.{ }^{\circledR}\right)$. The compartment specific surgical procedures have previously been described in detail, but are outlined below. In the anterior mesh implantation the polypropylene mesh arms were passed through the arcus tendineus fascia pelvis using four metal trocars in the anterior vaginal compartment (7).

In the posterior compartment, the mesh was placed through a transgluteal approach, and the two extension arms are fixated to the sacrospinous ligament (7). The combined mesh implantation technique was the combination of procedures in anterior and posterior mesh implants (7). In 22 women was used the uniformly sized and shaped polypropylene mesh; in 24 patients adjusting of the mesh to the local situation was necessary.

During the surgery, two major complications were monitored, i.e. significant blood loss (over $300 \mathrm{ml}$ ), and visceral organ injury (i.e. urinary bladder).

\section{Postoperative outcomes and evaluation of the complications}

A clinical examination and subjective symptom assessments were performed 1.5, 3, 6, 12 months after the surgery, and then annually (up to 7 years after the mesh implantation). The POP-Q quantification in the same time periods was used for the postoperative prolapse stage evaluation (recidives), as $0-\mathrm{I}$ was considered as an anatomical cure.

The evaluated complications in the mentioned time schedule included de novo stress urinary incontinence, de novo dyspareunia, mesh erosion (exposure), pelvic pain, urinary retention, and urinary tract infections.

\section{Risk factors of the mesh implantation}

According to the previously published studies, as risk factors were considered the lower age of the patient (below or equal to 65 years), higher body mass index (BMI) (overweight with BMI between 25 and $29.9 \mathrm{~kg} / \mathrm{m}^{2}$, or obesity with BMI $30 \mathrm{~kg} /$ $\mathrm{m}^{2}$ or more), sexual activity, smoking, other diseases, previous prolapse surgery, POP-Q stage $\geq 3$, and type of the mesh implantation (anterior, posterior, or both). All of these risk factors were evaluated for recidives, perioperative and postoperative complications.

\section{Statistics}

Statistical analyses were performed using SPSS17 software. Comparisons on data for dependent samples were performed using the Mann-Whitney U-test.

\section{Ethics}

All of the participants signed an informed consent for the mesh implantation surgery.

\section{Results}

\section{Per-surgery complications}

Forty six of 47 patients $(97.8 \%)$ had a successful mesh implantation (10 anterior, 22 posterior, and 14 combined). Complications of the mesh implantation occurred in 3 of 47 women (6.4\%) during the operation, i.e. blood loss over $300 \mathrm{ml}$ in one patient, and lesion of the bladder in two patients (in one of them the implantation was cancelled because of multiple bladder lesions). Detailed phenotype characterization of the 46 women with successful mesh implantation is shown in Table 1.

\section{Postoperative outcomes and evaluation of the complications}

The anatomic cure of the pelvic organ prolapse (POP-Q $\leq 1)$ was achieved in 45 from 46 (97.8\%) patients with implanted mesh at the time of 1.5 months after the surgery, in 43 from 46 (93.5\%) patients at 6 months after the surgery and in 41 from 46 (89.1\%) patients at 12 months after the surgery, respectively. The recidive of the pelvic organ prolapse was diagnosed altogether in 5 women; in one at 1.5 months after the implantation, in two at 3 months, and in other two individuals at 12 months, respectively. All five

Tab. 1. Phenotype characterization of the patients included to this study prior the mesh implantation.

\begin{tabular}{|c|c|c|c|c|c|c|c|c|c|c|}
\hline & & $\begin{array}{c}\text { Number } \\
\text { of } \\
\text { patients }\end{array}$ & $\begin{array}{c}\text { Age } \\
(\text { mean } \pm \text { SD }) \\
\text { years }\end{array}$ & $\begin{array}{c}\text { BMI } \\
(\text { mean } \pm \text { SD) } \\
\mathrm{kg} / \mathrm{m} 2\end{array}$ & $\begin{array}{c}\text { POP-Q stage } \\
(\text { mean } \pm \text { SD) }\end{array}$ & $\begin{array}{c}\text { Other diseases } \\
\text { No }(\%)\end{array}$ & $\begin{array}{l}\text { Smoking } \\
\text { No }(\%)\end{array}$ & $\begin{array}{c}\text { Any previous } \\
\text { surgery in the } \\
\text { pelvic region } \\
\text { No }(\%)\end{array}$ & $\begin{array}{c}\text { Hysterectomy } \\
\text { No }(\%)\end{array}$ & $\begin{array}{l}\text { Traditional } \\
\text { pelvic organ } \\
\text { prolapse sur- } \\
\text { gery No (\%) }\end{array}$ \\
\hline \multirow[t]{2}{*}{ Age } & $\leq 65$ years & 32 & $57.6 \pm 6.1$ & $28.2 \pm 3.3$ & $2.2 \pm 0.5$ & $15 / 32(46.9)$ & $2 / 32(6.3)$ & $32 / 32(100)$ & $30 / 32$ (93.7) & $21 / 36(65.6)$ \\
\hline & $>65 \mathrm{y}$ & 14 & $71.1 \pm 4.0$ & $28.0 \pm 2.7$ & $2.4 \pm 0.6$ & $9 / 14(64.3)$ & $0 / 14(0)$ & $14 / 14(100)$ & $14 / 14(100)$ & $8 / 14(57.1)$ \\
\hline \multicolumn{11}{|l|}{$\begin{array}{l}\text { Surgical } \\
\text { procedure }\end{array}$} \\
\hline & Anterior & 10 & $63.5 \pm 6.8$ & $27.9 \pm 3.0$ & $2.4 \pm 0.7$ & $5 / 10(50)$ & $0 / 10(0)$ & $10 / 1$ & $9 / 10(90)$ & $7 / 10(70)$ \\
\hline & Posterior & 22 & $60.3 \pm 7.5$ & $28.9 \pm 3.0$ & $2.2 \pm 0.4$ & $12 / 22(54.6)$ & $1 / 22(4.6)$ & $00)$ & $21 / 22(95.5)$ & $16 / 22(72.7)$ \\
\hline & Combined & 14 & $62.6 \pm 10.5$ & $27.1 \pm 3.1$ & $2.3 \pm 0.6$ & $7 / 14(50)$ & $1 / 14(7.1)$ & $14 / 14(100)$ & $14 / 14$ (100) & $6 / 14(42.9)$ \\
\hline Together & & 46 & $61.7 \pm 8.3$ & $28.1 \pm 3.1$ & $2.3 \pm 0.5$ & $24 / 46(52.2)$ & $2 / 46(4.3)$ & $46 / 46(100)$ & $44 / 46(95.7)$ & $29 / 46(63.0)$ \\
\hline
\end{tabular}




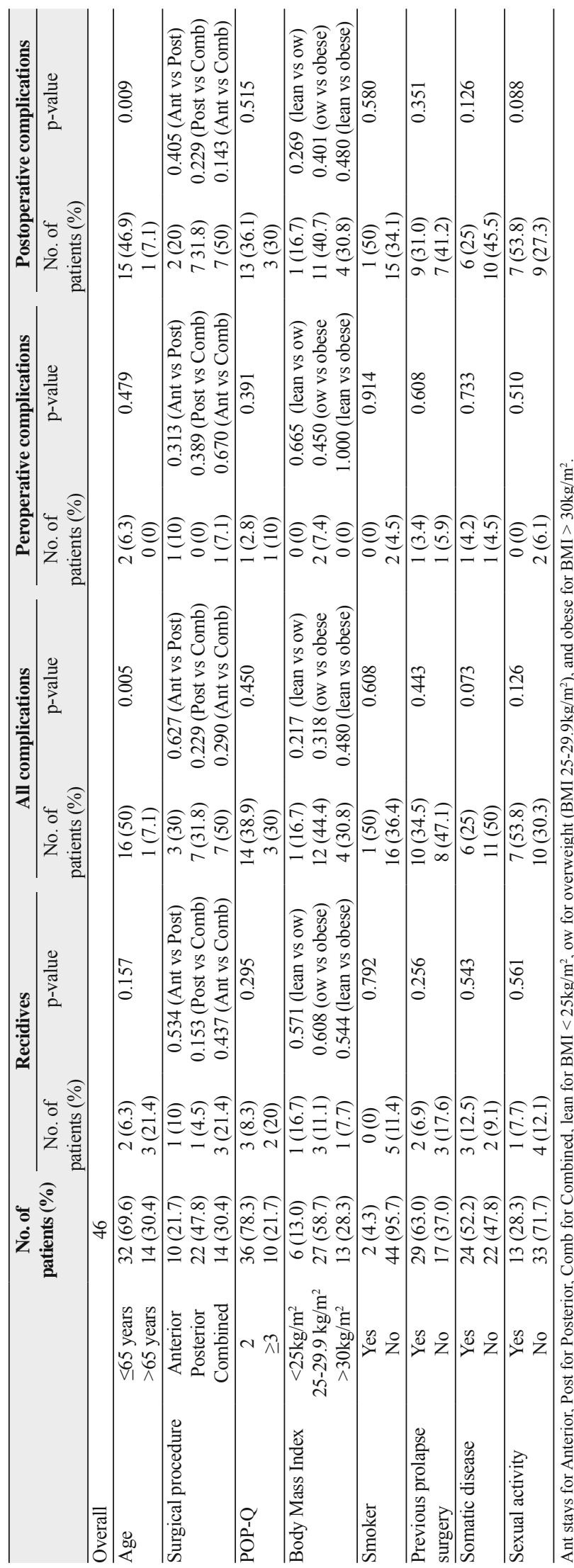

Tab. 2. Complications and recidives of the mesh implantation.

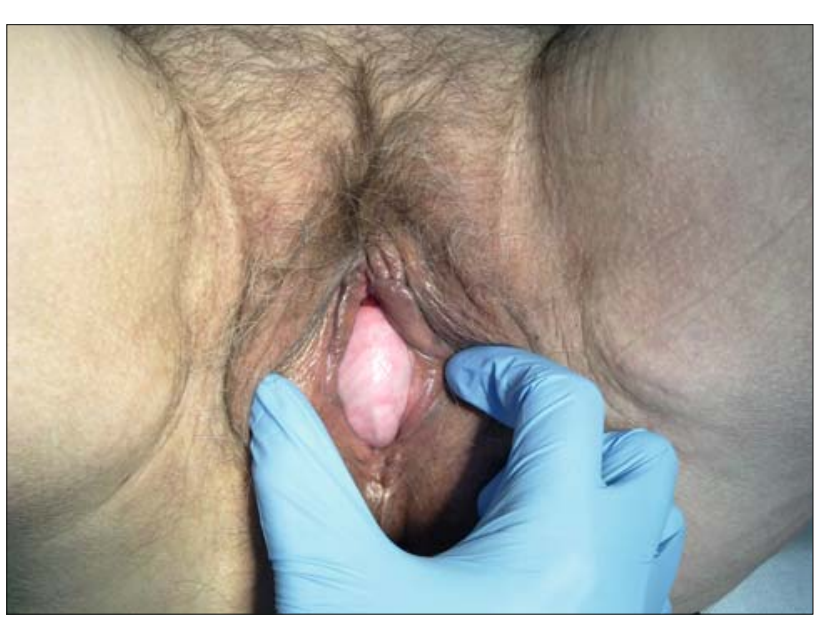

Fig. 1. Recidive of the pelvic organ prolapse of anterior compartment POP-Q2.

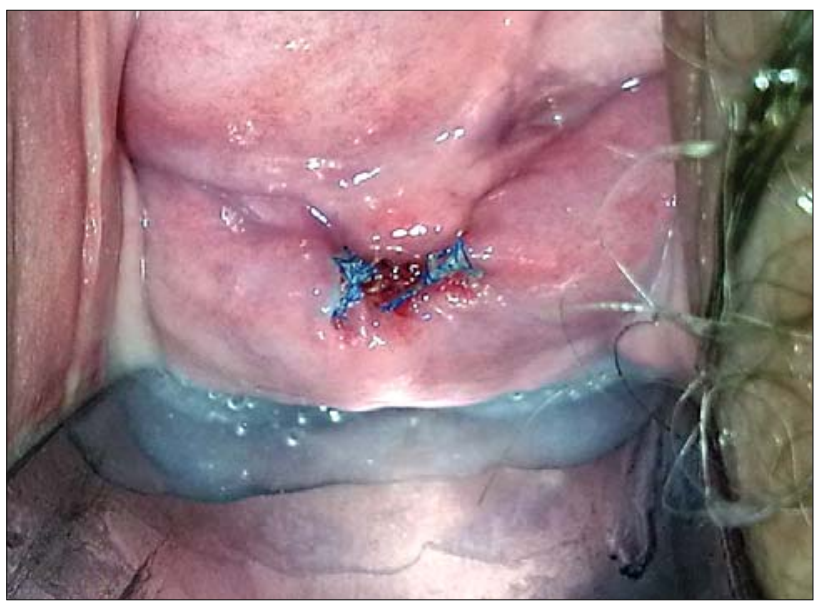

Fig. 2. Permanently unhealed exusure sized $5 \times 3 \mathrm{~mm}$ caused by protrusion of the mesh by a large postoperative hematoma.

patients with the recidive of the pelvic organ prolapse developed POP-Q stage II, and only one of them undergone resurgery of the prolapse - laparoscopic sacrocolpopexy with anatomic cure (to date -22 months after resurgery). In the remaining four women is the prolapse recidive stationary. Recidive of the pelvic organ prolapse is displayed on Figure 1.

During the follow-up (range 1-7 years) any of the monitored complications occurred in 16 of 46 women (34.8\%) (Tab. 2). All of the complications occured during the first 6 months after the surgery. The most common complication was de novo stress urinary incontinence in 7 of the 46 patients $(15.2 \%)$, followed by dyspareunia (in 3 of the 46 patients, i.e. $6.5 \%$ from the whole group, and $23.1 \%$ among sexually active individuals), pelvic pain (in 2 of 46 patients, i.e. $4.4 \%$ ), urinary tract infections (in 2 of 46 patients, i.e. $4.4 \%$ ), and exposures (in 2 of the 46 patients, i.e. $4.4 \%$ ). In one women the exposure cured spontaneously, the other sized $5 \times 3 \mathrm{~mm}$ was caused by protrusion of the mesh by a large postoperative hematoma and is permanently unhealed (Fig. 2). This patients is 67 years old, sexually inactive, and has no 
clinical symptoms due to the exposure. In our patients no urinary retention was recorded.

\section{Risk factors of the mesh implantation}

From the evaluated risk factors (i.e. age, body mass index, sexual activity, smoking, other diseases, previous prolapse surgery, POP-Q stage, and type of the mesh implantation), the only one with significant impact on the outcomes was the age at mesh implantation. Women $>65$ years had significantly lower risk of postoperative $(p=0.009)$ and peroperative + postoperative complications $(p=0.005)$ compare to the women aged $\leq 65$ years. All remaining differences were not significant (Tab. 2).

\section{Discussion}

Our study was focused on longitudinal follow-up after transvaginal mesh implantation of women with pelvic organ prolapse (POP-Q $\geq 2$ ) and a history of other surgery in the pelvic region accumulated over 7 years at the 2nd Department of Obstetrics and Gynecology in Bratislava, Slovakia. Forty six of 47 patients (97.8 \%) had a successful mesh implantation (10 anterior, 22 posterior, 14 combined). The anatomic cure of the pelvic organ prolapse (POP-Q $\leq 1)$ was achieved in 43 from 46 (93.5\%) patients with implanted mesh at the time of 6 months and in 41 from 46 (89.1\%) patients at 12 months after the surgery, respectively. Three patients had peroperative complication (including one individual with the unsuccessful mesh implantation), and 16 patients had postoperative complication $(7 \mathrm{x}$ de novo stress urinary incontinence, $3 \mathrm{x}$ dyspareunia, $2 \mathrm{x}$ exposures, $2 \mathrm{x}$ pelvic pain and $2 \mathrm{x}$ urinary tract infections). Significantly lower risk of complications was found in the group aged over 65 years compared to the younger patients $(\mathrm{p}=0.005)$.

This is the first study on the transvaginal mesh implantation including only women with the history of other surgery in the pelvic region, with long-term follow-up for up to 7 years.

\section{Strength and limitations}

The primary strength of this study is the homogeneity of the group of patients based on the strict inclusion criteria (i.e. previous surgery in the pelvic region). Majority of previously published studies did not include only high-risk individuals (according to the (6)). Strength is also that all mesh implantations were carried out by one team.

On the other hand, the strict criteria determined number of patients - 2nd Department of Obstetrics and Gynecology is the largest in Bratislava according to the number of patients with pelvic organ prolapse, and despite of this and period of 7 years only 47 of them met the strict criteria for the mesh implantation. This relatively low number of individuals could have an impact on the results, and significance of the data.

\section{Inclusion criteria}

Due to the FDA and Expert Committee Opinion, the pelvic organ prolapse vaginal mesh should be reserved for high-risk individuals (e.g. individuals with recurrent prolapse or with medical comorbidities) (6). Nevertheless, our inclusion criteria were based on the previous surgery in pelvic region, as this postoperative terrain is of high risk using the traditional prolapse surgery. Therefore, in the study individuals the mesh implantation was considered as favorite possibility for the cure of the prolapse.

\section{Comparison to other studies}

Compared to the other studies, we had high percentage of the anatomic cure of pelvic organ prolapse (POP-Q $\leq 1)$, i.e. $93.5 \%$ at the time of 6 months, and $89.1 \%$ at 12 months after the surgery, respectively. This number was lower in published uncontrolled trials (i.e. 80-90.5\%) (9-13), and also randomized trial (60.8\% at 12 moths) (5). These differences could be possibly explained by using the strict indication criteria. On the other hand, higher frequency of the peroperative complications in our study (6.4\% compared to $0-3.8 \%$ in published studies (3-4, 10) could be caused by difficulties of scarred tissue preparation in women with previous pelvic surgery. This could also explain higher frequency of de novo stress urinary incontinence in 7 of the 46 patients $(15.2 \%)$ observed in our study. The other monitored postoperative complications were similarly or less frequent than in other studies $(3-4,10)$. Nevertheless, de novo dyspareunia observed in only 3 of the 46 patients $(6.5 \%)$ is one of the lowest published so far. This fact could be also influenced by low number of sexually active patients (i.e. $28.3 \%$ ). The fact of higher complication risk seen in younger patients was already described by other authors (14).

\section{Implication for clinicians}

Outcomes of our study support the mesh implantation in women with pelvic organ prolapse fulfilling the strict indication criteria, particularly in individuals aged over 65 years.

\section{Conclusions}

This is the first study on the mesh implantation including 47 women with pelvic organ prolapse and the history of other surgery in the pelvic region only. The mesh implantation achieved high anatomic success rate ( $93.5 \%$ at 6 months after the surgery) with low risk of complications, particularly in the postoperative period. Thus, the outcomes of our study support the mesh implantation in women with pelvic organ prolapse fulfilling the strict indication criteria, particularly in individuals aged over 65 years.

\section{References}

1. Swift SE. The distribution of pelvic organ support in a population of female subjects seen for routine gynecologic health care. Am J Obstet Gynecol 2000; 183 (2): 277-285.

2. Olsen AL, Smith VJ, Bergstrom JO et al. Epidemiology of surgically managed pelvic organ prolapse and urinary incontinence. Obstet Gynecol 1997; 89 (4): 501-506.

3. Miedel A, Tegerstedt G, Morlin B, Hammarstrom M. A 5-year prospective follow-up study of vaginal surgery for pelvic organ prolapse. Int Urogynecol J Pelvic Floor Dysfunct 2008; 19 (12): 1593-1601. 
4. Altman D, Falconer C. Perioperative morbidity using transvaginal mesh in pelvic organ prolapse repair. Obstet Gynecol 2007; 109 (2 Pt 1):303-308.

5. Altman D, Vayrynen T, Engh ME et al. Anterior colporrhaphy versus transvaginal mesh for pelvic-organ prolapse. N Engl J Med 2011; 364 (19): 1826-1836.

6. Committee Opinion no. 513: vaginal placement of synthetic mesh for pelvic organ prolapse. Obstet Gynecol 2011; 118 (6): 1459-1464.

7. Holly I, Papcun P, Ferianec V, Holoman K. Implants in operative therapy in women with pelvic organ prolapse - two years of experience. Bratisl Lek Listy 2009; 110 (11): 692-696.

8. Bump RC, Mattiasson A, Bo K et al. The standardization of terminology of female pelvic organ prolapse and pelvic floor dysfunction. Am J Obstet Gynecol 1996; 175 (1): 10-17.

9. Nguyen JN, Burchette RJ. Outcome after anterior vaginal prolapse repair: a randomized controlled trial. Obstet Gynecol 2008; 111 (4): 891-898.
10. Carey M, Higgs P, Goh J et al. Vaginal repair with mesh versus colporrhaphy for prolapse: a randomised controlled trial. BJOG 2009; 116 (10): 1380-1386.

11. Ek M, Altman D, Falconer C et al. Effects of anterior trocar guided transvaginal mesh surgery on lower urinary tract symptoms. Neurourol Urodyn 2010; 29 (8): 1419-1423.

12. Nieminen $K$, Hiltunen $R$, Takala $T$ et al. Outcomes after anterior vaginal wall repair with mesh: a randomized, controlled trial with a 3 year follow-up. Am J Obstet Gynecol 2010; 203 (3): 235 e1-8.

13. Withagen MI, Milani AL, den Boon $\mathbf{J}$ et al. Trocar-guided mesh compared with conventional vaginal repair in recurrent prolapse: a randomized controlled trial. Obstet Gynecol 2011; 117 (2 Pt 1): 242-250.

14. Elmer C, Altman D, Engh ME et al. Trocar-guided transvaginal mesh repair of pelvic organ prolapse. Obstet Gynecol 2009; 113 (1): 117-126.

Received March 10, 2014. Accepted April 28, 2014. 